\title{
RRM2B-Mediated Regulation of Mitochondrial Activity and Inflammation under Oxidative Stress
}

\author{
Er-Chieh Cho, ${ }^{1,2}$ Mei-Ling Kuo, ${ }^{3}$ Jia-hui Cheng, ${ }^{1}$ Yu-Chi Cheng, ${ }^{4}$ Yi-Chen Hsieh, ${ }^{5}$ \\ Yun-Ru Liu, ${ }^{6}$ Rong-Hong Hsieh, ${ }^{4}$ and Yun Yen ${ }^{7}$ \\ ${ }^{1}$ Department of Clinical Pharmacy, School of Pharmacy, College of Pharmacy, Taipei Medical University, Taipei 110, Taiwan \\ ${ }^{2}$ Master Program for Clinical Pharmacogenomics and Pharmacoproteomics, School of Pharmacy, Taipei Medical University, \\ Taipei 110, Taiwan \\ ${ }^{3}$ Department of Molecular Pharmacology, Beckman Research Institute, City of Hope, Duarte, CA 91010, USA \\ ${ }^{4}$ School of Nutrition and Health Sciences, College of Public Health and Nutrition, Taipei Medical University, Taipei 110, Taiwan \\ ${ }^{5}$ Ph.D. Program for Neural Regenerative Medicine, College of Medical Science and Technology, Taipei Medical University, \\ Taipei 110, Taiwan \\ ${ }^{6}$ Joint Biobank, Office of Human Research, Taipei Medical University, Taipei 110, Taiwan \\ ${ }^{7}$ Graduate Institute of Cancer Biology and Drug Discovery, College of Medical Science and Technology, Taipei Medical University, \\ Taipei 110, Taiwan
}

Correspondence should be addressed to Er-Chieh Cho; echo@tmu.edu.tw and Yun Yen; yyen@tmu.edu.tw

Received 31 December 2014; Revised 25 March 2015; Accepted 25 March 2015

Academic Editor: Sandra Helena Penha Oliveira

Copyright (C) 2015 Er-Chieh Cho et al. This is an open access article distributed under the Creative Commons Attribution License, which permits unrestricted use, distribution, and reproduction in any medium, provided the original work is properly cited.

RRM2B is a critical ribonucleotide reductase (RR) subunit that exists as p53-inducible and p53-dependent molecule. The p53independent regulation of RRM2B has been recently studied, and FOXO3 was identified as a novel regulator of RRM2B. However, the p53-independent regulation of RRM2B, particularly under oxidative stress, remains largely unknown. In this study, we investigated the role of RRM2B underoxidative stress-induced DNA damage and further examined the regulation of mitochondrial and inflammatory genes by RRM2B. Our study is the first to report the critical role of RRM2B in mitochondrial homeostasis and the inflammation signaling pathway in a p53-independent manner. Furthermore, our study provides novel insights into the role of the RR in inflammatory diseases.

\section{Introduction}

Ribonucleotide reductase (RR) catalyzes the conversion of ribonucleoside diphosphates into deoxyribonucleoside diphosphates, playing essential roles in DNA synthesis and repair in humans and influencing vital cellular mechanisms [1-5]. The molecular regulations of the three known RR subunits, RRM1, RRM2, and RRM2B (also called p53R2), have long been studied and reported by our and other groups [6-15]. Previous research has established that RRM2B, a p53inducible RR subunit, plays vital roles in DNA repair, cell cycle modulation, mitochondrial DNA (mtDNA) synthesis, metastasis suppression, and oxidative stress resistance $[1$, $6,7,9,16-19]$. Mutation or absence of $R r m 2 b$ in humans results in defective mtDNA, and severe mtDNA depletion has been observed in Rrm2b-/- animals $[9,16]$. In cells, RRM2B can be regulated by p53 and p73, a p53 family member. In addition, RRM2B regulates the p53-dependent cell cycle for DNA damage [6]. RRM2B can suppress the metastasis and proliferation of different cancer cells [20]. A study on a Rrm $2 b$-knockout animal model indicated that the intactness of the RRM2B subunit is critical for maintaining chromosomal stability and that the loss of RRM2B results in plasmacytic neoplasms [21]. Furthermore, RRM2B expression is correlated with improved survival in some cancers, whereas a more progressive phenotype of certain other cancers complicates the RRM2B regulatory pathway [22-24].

Several reports have suggested that RRM2B regulates critical cellular mechanisms irrespective of the p53 status. First, although the p53-mediated RRM2B induction is inhibited in 
the p53-deficient mouse embryonic fibroblasts cells, it has been observed that RRM2B is expressed at basal levels [16]. Furthermore, a high RRM2B expression has been observed in various p53-deficient cancer cells, and it continues to influence mitochondrial functions irrespective of the p53 status, suggesting that RRM2B-mediated mitochondrial homeostasis is independent of functional p53, and other factors are involved in RRM2B regulation $[25,26]$. The findings of our recent study are consistent with those of the aforementioned studies, in which the tumor suppressor FOXO3 binds to the RRM2B promoter and activates RRM2B transcription in a p53-independent manner under physiological conditions [27].

RRM2B is a unique member among the RR subunits that can resist reactive oxygen species (ROS) $[8,19,25,28$, 29]. However, little is known about the detailed mechanisms and the functional regulations of RRM2B under oxidative stress. We studied the functional regulations of RRM2B in cancer cells under oxidative stress and observed that RRM2B plays a crucial role in the regulation of mitochondrial and inflammation pathways in a p53-independent manner.

\section{Materials and Methods}

2.1. Cell Lines, Plasmids, and Stable Cell Line Production. Cells from ATCC were cultured in DMEM medium containing fetal bovine serum (10\%) and penicillin/streptomycin (1\%) incubated at $37^{\circ} \mathrm{C}$ with $5 \% \mathrm{CO}_{2}$. RRM2B overexpression and shRNA plasmids were described before [28]. Stable cell lines were established by infection and selection as described [28].

2.2. Immunofluorescence. The assay was performed as described [30]. In brief, H1299 cells were seeded on coverslips, fixed, permeabilized, stained with primary antibodies, antiRRM2B (Rockland) and $\gamma$-H2AX (Active Motif), and secondary antibodies (Invitrogen), washed, and then mounted with DAPI (Invitrogen) onto the slides. Samples were analyzed by immunofluorescence microscope system, and the scale bars indicate $10 \mu \mathrm{M}$.

2.3. Western Blot Analysis and Cytoplasmic/Nuclear Protein Extraction. Cell extracts were prepared and analyzed as described before [31]. Antibodies used in this assay were RRM2B (Rockland), $\gamma$-H2AX (Active Motif), GAPDH (Santa Cruz and GeneTex), VDAC1, COX4, p-NFאB, p-p38, and p$\mathrm{I} \kappa \mathrm{B}$ (GeneTex). The cytoplasmic/nuclear extraction kit was applied to separate cytoplasmic and nuclear fractions as described in the manufactory protocol (TOOLS) before processing to the western on phospho antibodies. Quantitative protein expression relative to GAPDH was analyzed by Image J where applicable.

2.4. Mitochondrial Mass Measurement. Cells were treated with $\mathrm{H}_{2} \mathrm{O}_{2}$, harvested, washed with PBS, and stained with MitoTracker Green probes (Invitrogen) of $50 \mathrm{nM}$ in serumfree medium for 30 mins. Cells were washed with PBS and analyzed by FACSCanto II cytometer. Fluorescent intensity was analyzed by FACSCanto II program.
2.5. Real-Time PCR Analysis. Total RNA was isolated by Trizol, and then RNA was reverse transcribed (Quanta) to obtain cDNA for PCR [32]. cDNA was subjected to real-time PCR using the SYBR Green PCR reagents kit (Stratagene) as described [33]. For quantitation of mtDNA copy number, $N d 1$ gene level was quantified by q-PCR and normalized to $\beta$ Actin. Due to the space limitation, primers are provided upon request.

2.6. Statistical Methods. The statistical analysis was done as described [27]. In brief, Student's $t$-test was used for $P$ value calculation, and the star ${ }^{*}$ stands for $P<0.05$. The results shown in this paper are representative data.

\section{Results}

3.1. Impact of $\mathrm{RRM} 2 \mathrm{~B}$ on $\mathrm{H}_{2} \mathrm{O}_{2}$-Mediated DNA Damage Resistance in p53-Deficient Cells. Although it is a known fact that RRM2B can resist ROS, little is known about the detailed regulatory mechanisms. We hypothesized that the oxidative resistance of RRM2B is independent of p53. Stable $\mathrm{H} 1299$ cell lines without the functional p53 protein containing overexpressed RRM2B or RRM2BshRNA and their respective controls were included in this study, as described previously, [27] and RRM2B expression was evaluated using Western blot analysis (Figure 1(a)) and an immunofluorescence assay (Figure 1(b)).

For investigating $\mathrm{RRM} 2 \mathrm{~B}$ regulation under oxidative stress, hydrogen peroxide $\left(\mathrm{H}_{2} \mathrm{O}_{2}\right)$, a type of oxidative stressinducing ROS, was used as the source of oxidative stress in this study. Cells were treated with $\mathrm{H}_{2} \mathrm{O}_{2}$ for 2 hours and fixed for immunofluorescence staining, in which $\gamma$ $\mathrm{H} 2 \mathrm{AX}$ foci induction indicated DNA damage. The results suggested that stronger $\gamma$-H2AX signals were induced by cells expressing RRM2BshRNA than by the control cells (Figure 2(a)), suggesting that greater DNA damage was associated with lower RRM2B expression in cells.

For the Western blot analysis, we performed the experiment at different time intervals, and the $\gamma$-H2AX signals on the blots were quantified relative to the GAPDH activity for quantitative estimation and analysis. Consistent RRM2B depletion was observed, which was correlated with the induction of $\gamma$ - $\mathrm{H} 2 \mathrm{AX}$ signals under $\mathrm{H}_{2} \mathrm{O}_{2}$ treatment (Figure 2(b)). Similarly, we observed low $\gamma$-H2AX foci signals under $\mathrm{H}_{2} \mathrm{O}_{2}$ treatment in RRM2B overexpressed cells (Figure 2(c)). In summary, the results suggested that RRM2B plays a protective role in securing cells against oxidative stressinduced DNA damage in a p53-independent manner.

\subsection{RRM2B-Mediated Impact on Mitochondrial Regulation} under Oxidative Stress. Next, we examined the role of RRM2B in regulating mitochondrial proteins under oxidative stress in p53-deficient H1299 cells. Only stable cells expressing RRM2BshRNA or those acting as control that were subjected to or excluded from $\mathrm{H}_{2} \mathrm{O}_{2}$ treatment were included in this study. The cells were then harvested for Western blot analysis, in which the expression of mitochondrial proteins, voltage-dependent anion channel 1 (VDAC1), and cytochrome c oxidase subunit IV (COX4) [34-36] 


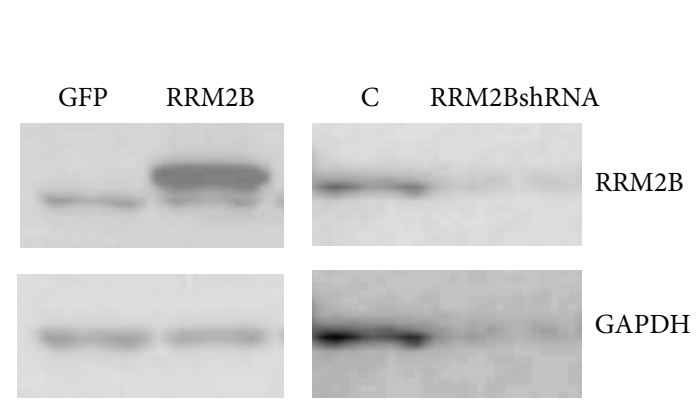

(a)

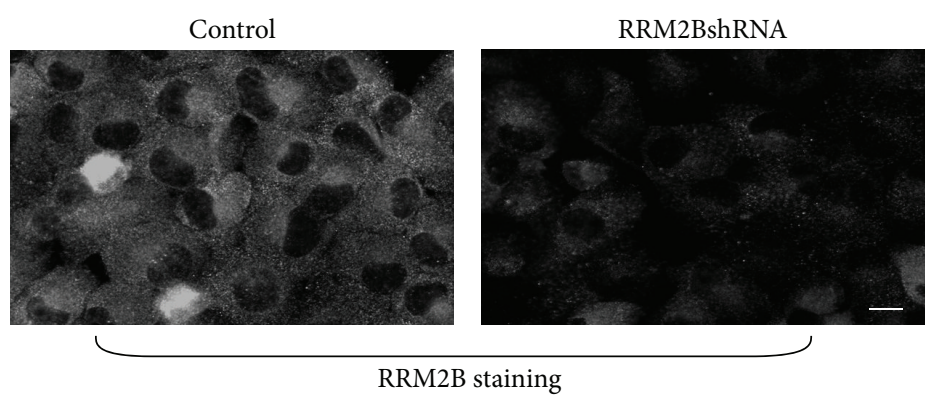

(b)

Figure 1: Expression of RRM2B in the H1299 stable cell lines. H1299 stable cell lines were selected as described in the Section 2. Cells were harvested for (a) Western blot analysis or (b) fixed for immunofluorescence assay. Anti-RRM2B and GAPDH were applied in the assays.

were examined along with RRM2B expression and GAPDH activity (Figure 3(a)(i)). In addition, the relative expression levels of VDAC1 and COX4 were analyzed and are shown in Figure 3(a)(ii). The results suggested that the expression levels of both proteins, particularly $\mathrm{VDACl}$, were more pronounced under $\mathrm{H}_{2} \mathrm{O}_{2}$ treatment, and the expression levels decreased on RRM2B downregulation, confirming that RRM2B regulated the mitochondrial content (Figure 3(a)).

To further understand the impact of RRM $2 B$ on the mitochondria, the mitochondrial mass was measured. FACS was performed using a MitoTracker Green probe for detecting the mitochondrial mass in stable H1299 cells under $\mathrm{H}_{2} \mathrm{O}_{2}$ treatment. Under oxidative stress, a significant decrease was observed in the relative mitochondrial mass of the cells expressing RRM2BshRNA, indicating the protective role of RRM2B in mitochondrial homeostasis (Figure 3(b)(i)), which is in agreement with our previous finding [25]. The representative examples of the intensity of MitoTracker on FACS are shown in Figure 3(b)(ii).

Moreover, quantitative PCR was used for measuring the relative mtDNA copy number. The relative copy number of the NADH dehydrogenase subunit $1(N d 1)$, a mitochondrial gene [37], under $\mathrm{H}_{2} \mathrm{O}_{2}$ treatment was measured using quantitative PCR. Low RRM2B expression resulted in a low Nd1 copy number (Figure 3(c)), suggesting that, under oxidative stress, RRM2B exerts protective effects on the mtDNA content.

The results establish that the RRM2B pathway affects mitochondrial homeostasis under oxidative stress, which is independent of the action of functional p53.

\subsection{RRM2B Affects the Inflammation Pathway under Oxida-} tive Stress. Oxidative stress mediated by $\mathrm{H}_{2} \mathrm{O}_{2}$ triggers the inflammation pathway $[38,39]$, and human inflammatory diseases have long been associated with NF- $\kappa$ B or p38 signaling or both [40-42]. However, the role of RRM2B in the regulation of inflammation has not been investigated. $\mathrm{NF}-\kappa \mathrm{B}$ and $\mathrm{p} 38$ are complex pathways regulating various cellular mechanisms [41, 43-46]. The NF- $\kappa$ B pathway can be activated by various proinflammatory cytokines and is therefore considered a proinflammatory signaling pathway. In addition, extracellular stimuli such as UV light, growth factors, and inflammatory cytokines result in p38 activation. In this study, we investigated the functional role of RRM2B in oxidative stress-mediated NF- $\kappa$ B and p 38 signaling. Stable H1299 control or RRM2BshRNA cells were treated with $\mathrm{H}_{2} \mathrm{O}_{2}$ and harvested 2 hours later, and the lysates were separated into cytoplasmic and nucleolus fractions. Western blot analysis was performed using phosphorylated NF- $\kappa \mathrm{B}$, phosphorylated $\mathrm{I} \kappa \mathrm{B}$, and phosphorylated $\mathrm{p} 38$ antibodies for identifying NF- $\kappa$ B and p38 activation in cells (Figure 4).

The results showed that nuclear NF- $\kappa \mathrm{B}$ and p 38 signaling on $\mathrm{H}_{2} \mathrm{O}_{2}$ treatments were more pronounced in the cells expressing RRM2BshRNA than in the control cells (Figure 4), suggesting that a stronger inflammation signal was induced by RRM2B depletion.

In summary, the findings suggest for the first time that RRM2B can functionally regulate the mitochondrial and inflammatory pathway and that these RRM2B-mediated regulations are independent of p53. Figure 5 illustrates a model summarizing the current findings of RRM2B regulation, suggesting that, under oxidative stress, RRM2B plays critical roles in the upregulation of genes involved in the mitochondrial and inflammation pathways.

\section{Conclusion and Discussion}

RRM2B is a unique member of the RR enzyme that exhibits anti-ROS potential. It was demonstrated that RRM2B suppressed ROS activation mediated by oxidative stress and is highly induced in a p53-dependent manner during senescence [28]. In our recent study, $\mathrm{FOXO} 3$ was observed to be a novel regulator of RRM2B [27]. In this study, the critical role of RR2MB in regulation of mitochondrial and inflammation pathways under oxidative stress in a p53independent manner was reported for the first time.

RRM2B plays critical roles in vital cellular mechanisms such as DNA replication, and low RRM2B expression sensitizes cancer cells under various stresses, and therefore studies have suggested that low RRM2B expression can potentially be considered a chemosensitizer for cancer treatment $[2,47$, 48]. In this study, H1299 cells were subjected to oxidative stress, and the induced $\gamma \mathrm{H} 2 \mathrm{AX}$ foci signals were stronger in RRM2BshRNA cells, indicating that the chemosensitivity 


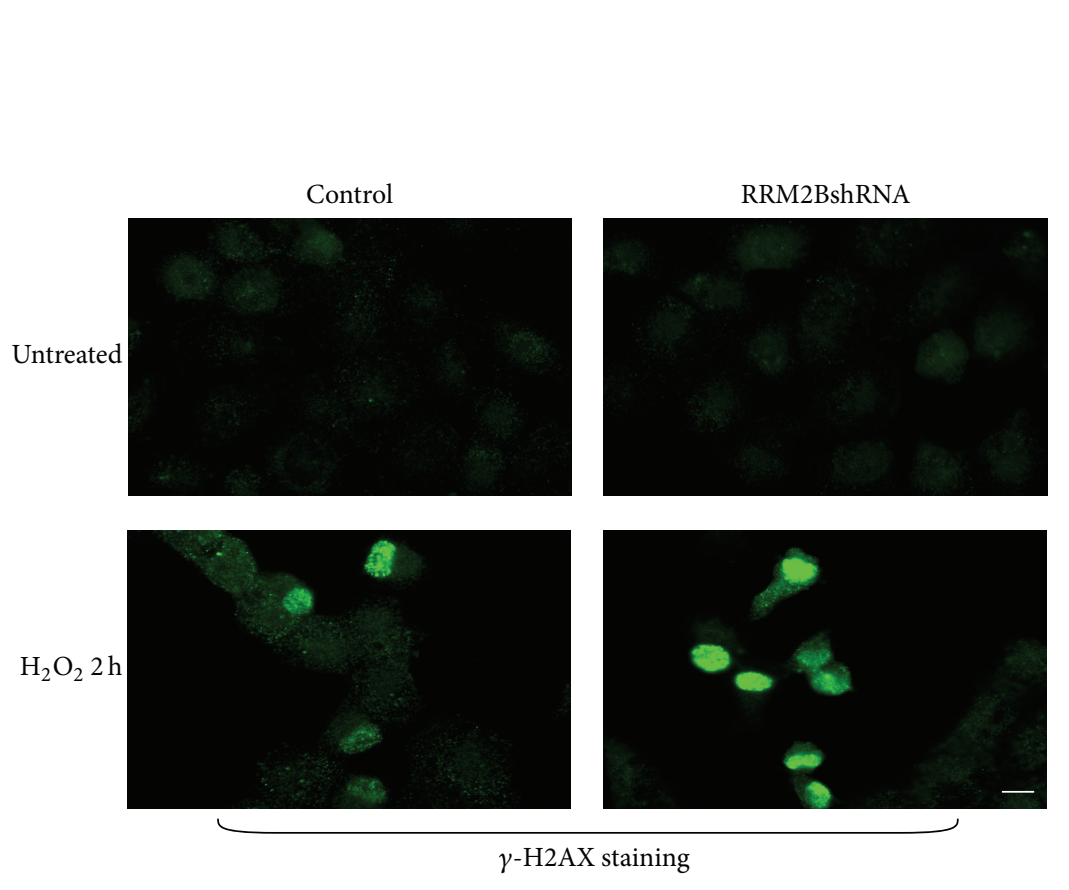

(a)

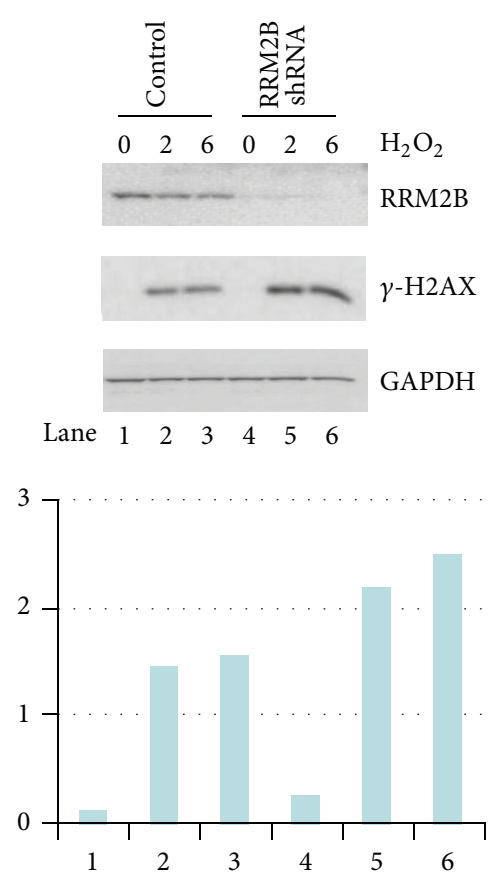

(b)

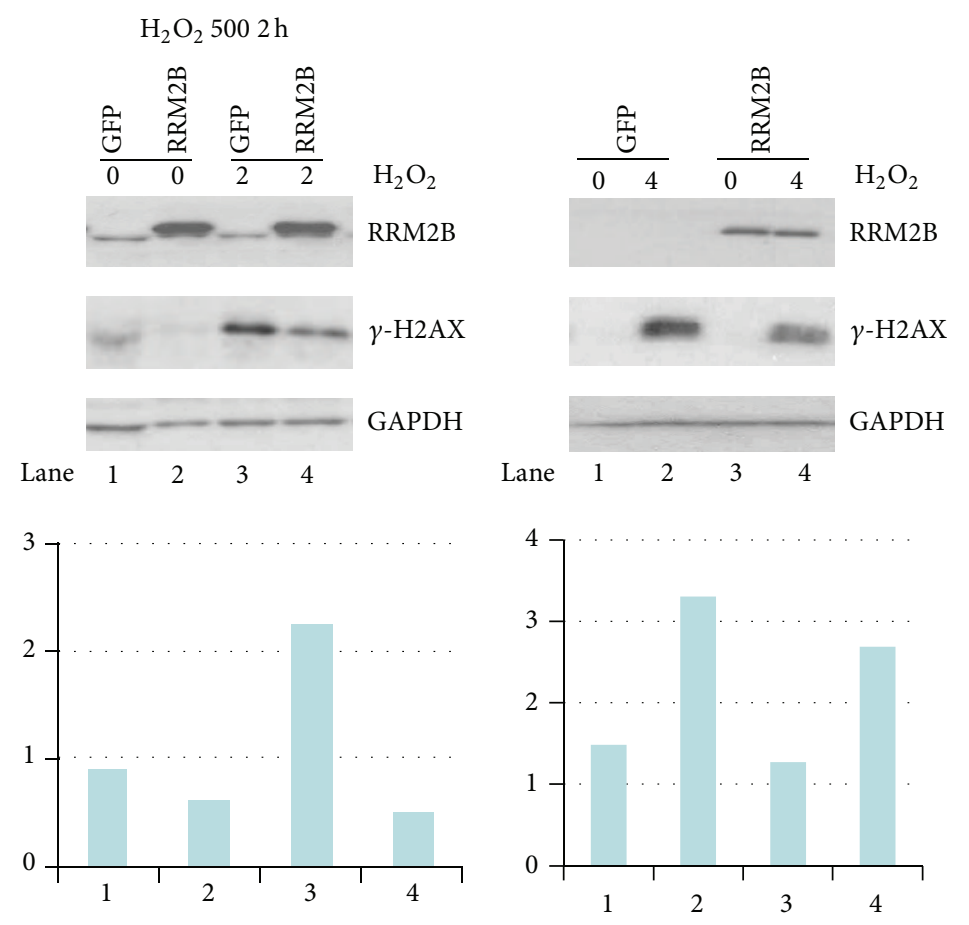

(c)

Figure 2: RRM2B functions in $\mathrm{H}_{2} \mathrm{O}_{2}$-mediated DNA damage resistance in p53-deficient cells. (a) $\mathrm{H} 1299$ stable cells were treated with $500 \mu \mathrm{M} \mathrm{H}_{2} \mathrm{O}_{2}$ for 2 hours where applicable for immunofluorescence assay analysis with anti- $\gamma$ - $\mathrm{H} 2 \mathrm{AX}$ antibody. (b)-(c) Two pairs of cell lines expressing control vectors, RRM2BshRNA and RRM2B expressing vector, were treated with $\mathrm{H}_{2} \mathrm{O}_{2}$ and harvested at different time points for Western blots analysis with indicated antibodies. $(n=2)$ Image J was used to normalize $\gamma$-H2AX expression to GAPDH. 

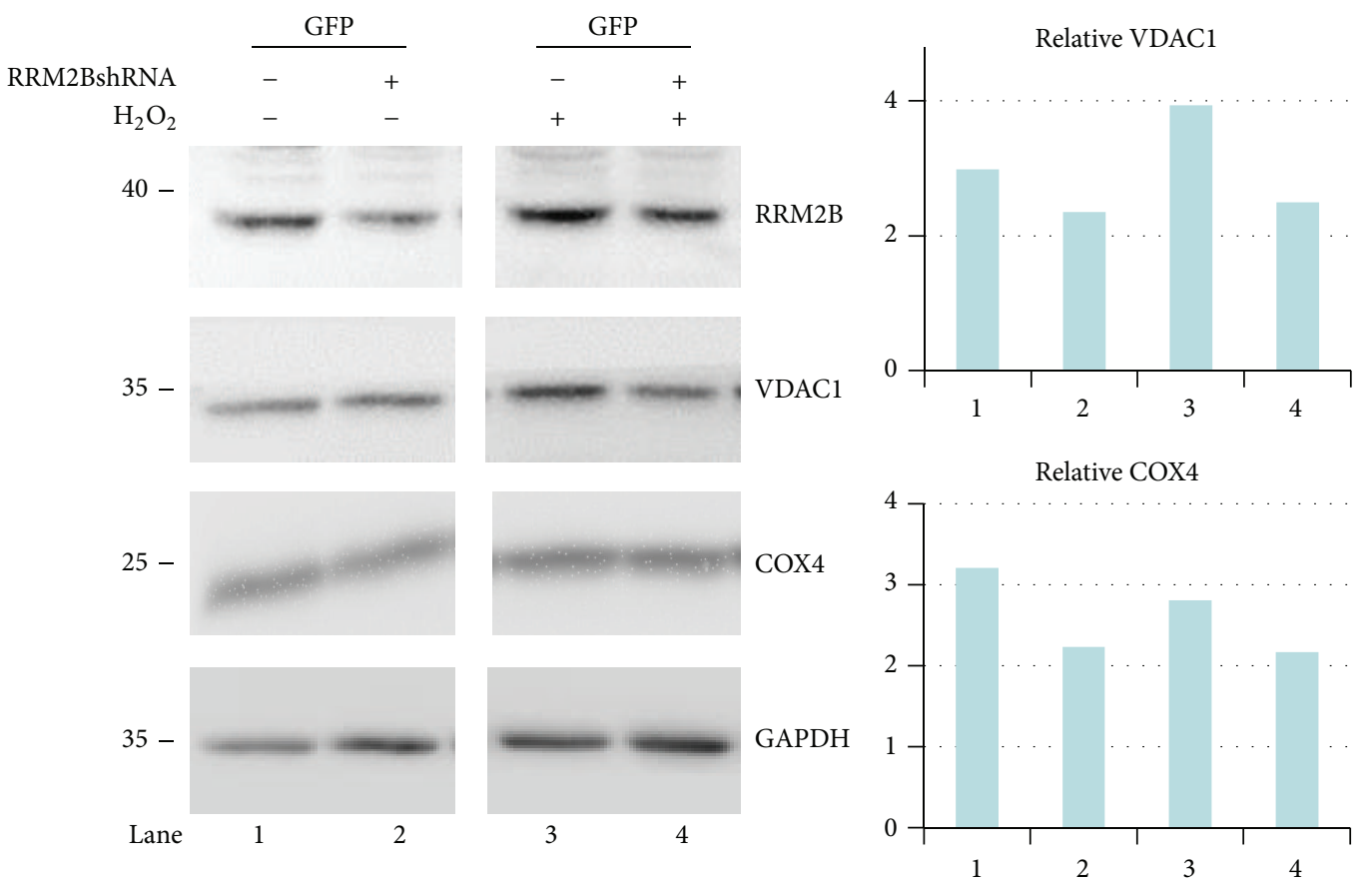

(i)

(ii)

(a)

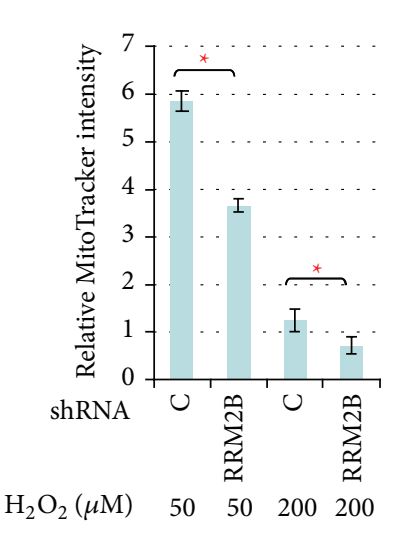

(i)

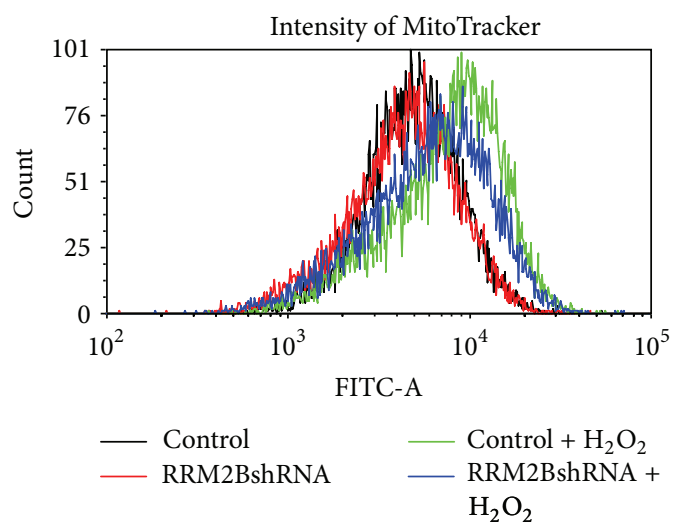

(ii)

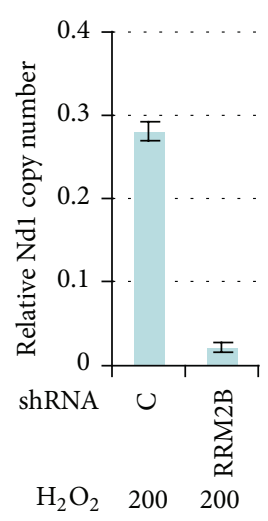

(c)

(b)

FIGURE 3: RRM2B impacts on mitochondrial homeostasis through regulating mitochondrial genes and copy number under oxidative stress. (a) Cells were treated with $\mathrm{H}_{2} \mathrm{O}_{2}$ and harvested for Western blot analysis using RRM2B and mitochondrial proteins VDAC1 and COX4 antibodies. GAPDH served as control. Image J was used to normalize VDAC1 and COX4 expression relative to GAPDH, and the normalized figures were shown in 3(a)(ii). (b)(i) H1299 stable cells treated with indicated concentrations of $\mathrm{H}_{2} \mathrm{O}_{2}$ were harvested 24 hours later for FACS analysis. The detection of MitoTracker was described in Section 2. The intensity of the MitoTracker Green signal relative to untreated control cells is shown here (means \pm SEM, $n=2$ ). (b)(ii) Cells were treated with $\mathrm{H}_{2} \mathrm{O}_{2}$ and harvested at 24-hour time point and underwent FACS analysis. MitoTracker Green probe was used for cell staining, and this figure shows the MitoTracker intensity of the representative data from (b)(i). (c) Cells were treated with $200 \mu \mathrm{M} \mathrm{H}_{2} \mathrm{O}_{2}$ and harvested 24 hours later for Q-PCR analysis. Expression of normalized Nd1 relative to untreated control cells is shown (means \pm SEM, $n=2$ ).

may generate equal outcomes in p53-deficient cancer cells. Additional therapeutical applications remain to be uncovered.

As mentioned, $R R M 2 B$ mutation results in severe mtDNA depletion [9]. In this study, we further demonstrated that the presence of RRM2B affects mitochondrial protein expression.
Mitochondrial mass was damaged under low RRM2B expression and was further destroyed under oxidative stress. Findings of previous studies and the data in the present study indicate that the intactness of RRM2B is critical for complete functioning of the mitochondria, despite the presence of functional p53. 


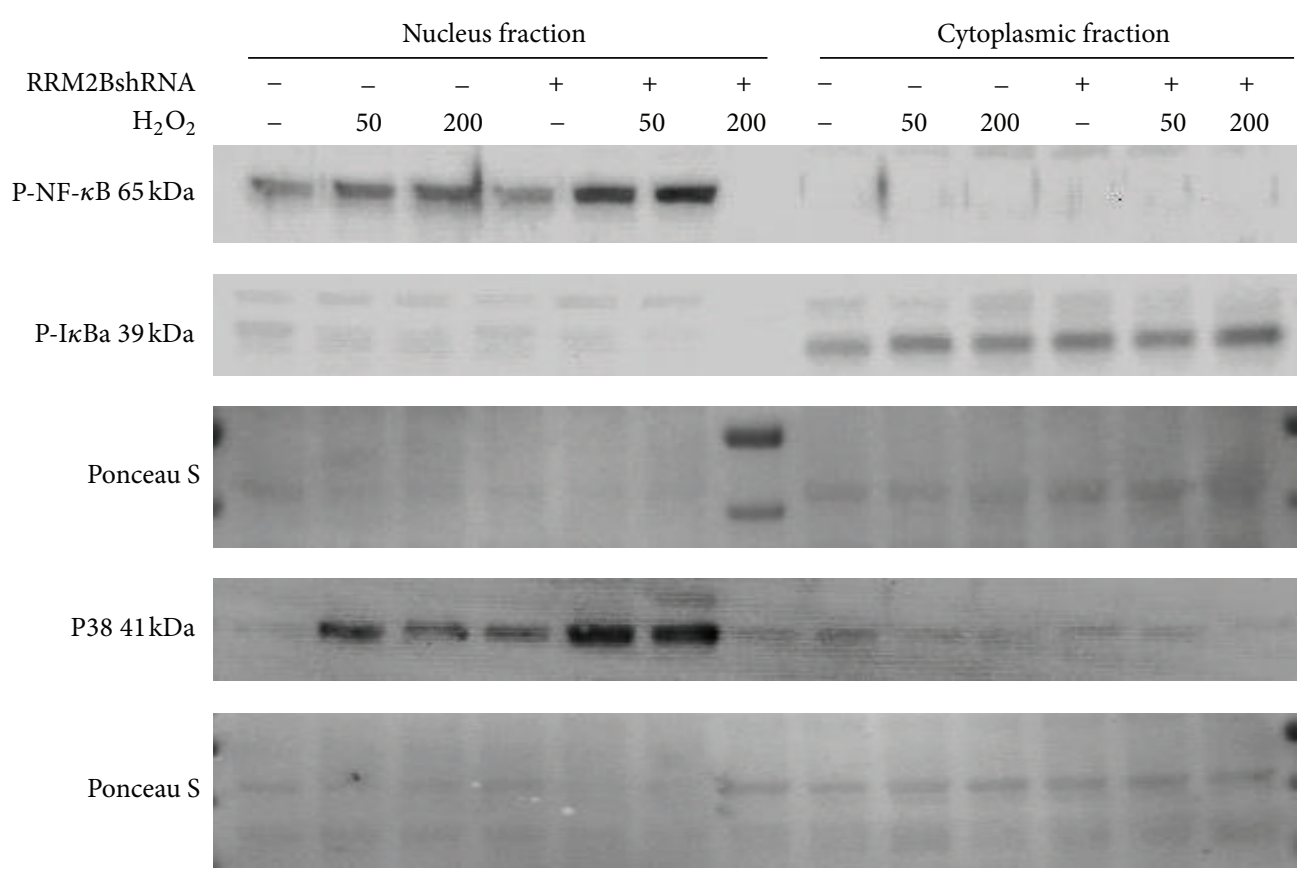

FIGURE 4: RRM2B regulates inflammatory signaling pathway under oxidative stress. (a) Stable cells were treated with 50 or $200 \mu \mathrm{M} \mathrm{H}_{2} \mathrm{O}_{2}$ and harvested 2 hours later, and the lysates were separated into nuclear and cytoplasmic fractions for Western blot analysis. Antibodies against phosphorylated NF- $\kappa \mathrm{B}$, phosphorylated $\mathrm{I} \kappa \mathrm{B}$, and phosphorylated $\mathrm{p} 38$ were applied, and GAPDH was used as loading control.

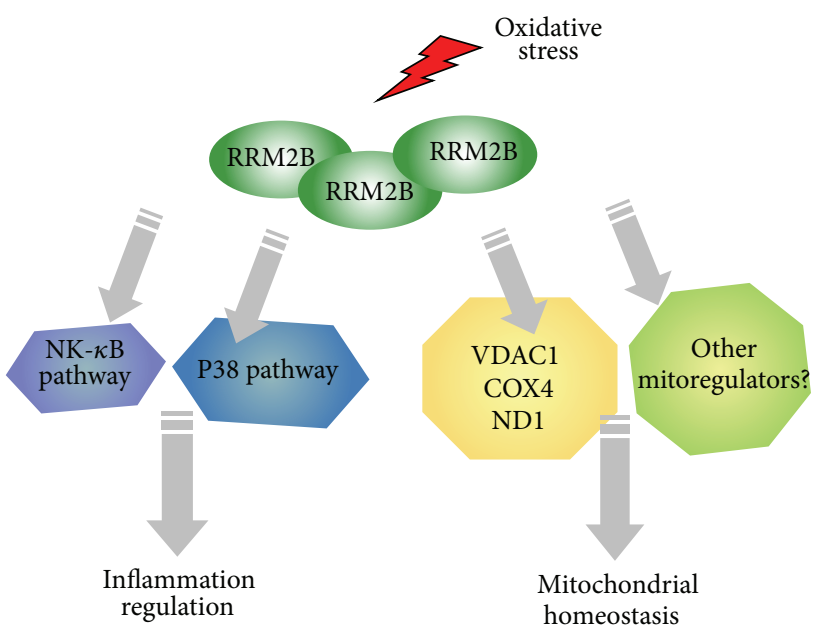

FIGURE 5: A model of RRM2B-mediated regulation of inflammatory $\mathrm{NF}-\kappa \mathrm{B}$ and $\mathrm{p} 38$ pathways and mitochondrial pathways upon oxidative stress.

Oxidative stress has been shown to have a strong association with inflammation pathways. Moreover, our previous study using RRM2B-knockout animal models suggested that RRM2B is critical in maintaining chromosomal stability and preventing chronic inflammation-associated tumorigenesis [21]. In this study, we demonstrated that the NF- $\kappa \mathrm{B}$ and $\mathrm{p} 38$ signaling pathways were upregulated by oxidative stress, particularly under low RRM2B conditions, which is in agreement with our previous finding, suggesting that RRM2B is crucial in preventing chronic inflammation and acts by inhibiting the NF- $\kappa$ B and p38 pathways.
Both NF- $\kappa$ B and p38 signaling pathways affect vital cellular regulatory mechanisms that include inflammation and apoptosis $[46,49]$. Therefore, accompany with our data, the RRM2BshRNA cells could potentially trigger stronger inflammation and apoptosis signals under DNA damage conditions. Recently, more complex roles of NF- $\kappa \mathrm{B}$ have been suggested in NF- $\kappa \mathrm{B}$ activation in pro- and anti-inflammation processes and pro- and antiapoptosis $[49,50]$, which may depend on the nature of the model systems. The status of p53 further increased the complexity. Our current study provides new insights into the role of RR in inflammatory diseases, and the intriguing regulation mechanisms of RRM2B under oxidative stress in inflammation remain to be explored.

\section{Conflict of Interests}

The authors have no conflict of interests to declare.

\section{Acknowledgments}

The authors are grateful to the Ministry of Science and Technology (MOST 103-2320-B-038-006-MY2), Taipei Medical University (TMUTOP103004-2), and Comprehensive Cancer Center of Taipei Medical University (funding from Health and Welfare Surcharge of Tobacco Products, MOHW104TDU-B-212-124-001), Taiwan, for supporting this study.

\section{References}

[1] H. Tanaka, H. Arakawa, T. Yamaguchi et al., "A ribonucleotide reductase gene involved in a p53-dependent cell-cycle checkpoint for DNA damage," Nature, vol. 404, no. 6773, pp. 42-49, 2000 . 
[2] J. Shao, B. Zhou, B. Chu, and Y. Yen, "Ribonucleotide reductase inhibitors and future drug design," Current Cancer Drug Targets, vol. 6, no. 5, pp. 409-431, 2006.

[3] N. M. F. S. A. Cerqueira, P. A. Fernandes, and M. J. Ramos, "Ribonucleotide reductase: a critical enzyme for cancer chemotherapy and antiviral agents," Recent Patents on Anti-Cancer Drug Discovery, vol. 2, no. 1, pp. 11-29, 2007.

[4] Y. Aye, M. Li, M. J. C. Long, and R. S. Weiss, "Ribonucleotide reductase and cancer: biological mechanisms and targeted therapies," Oncogene, 2014.

[5] E. Guarino, I. Salguero, and S. E. Kearsey, "Cellular regulation of ribonucleotide reductase in eukaryotes," Seminars in Cell and Developmental Biology, vol. 30, pp. 97-103, 2014.

[6] K. Nakano, E. Balint, M. Ashcroft, and K. H. Vousden, "A ribonucleotide reductase gene is a transcriptional target of p53 and p73," Oncogene, vol. 19, no. 37, pp. 4283-4289, 2000.

[7] B. Zhou, X. Liu, X. Mo et al., "The human ribonucleotide reductase subunit hRRM2 complements p53R2 in response to UVinduced DNA repair in cells with mutant p53," Cancer Research, vol. 63, no. 20, pp. 6583-6594, 2003.

[8] L. Xue, B. Zhou, X. Liu et al., "Structurally dependent redox property of ribonucleotide reductase subunit p53R2," Cancer Research, vol. 66, no. 4, pp. 1900-1905, 2006.

[9] A. Bourdon, L. Minai, V. Serre et al., "Mutation of RRM2B, encoding p53-controlled ribonucleotide reductase ( $\mathrm{p} 53 \mathrm{R} 2)$, causes severe mitochondrial DNA depletion," Nature Genetics, vol. 39, no. 6, pp. 776-780, 2007.

[10] P. Smith, B. Zhou, N. Ho et al., " $2.6 \AA$ X-ray crystal structure of human p53R2, a p53-inducible ribonucleotide reductase," Biochemistry, vol. 48, no. 46, pp. 11134-11141, 2009.

[11] M. Xie, Y. Yen, T. K. Owonikoko et al., "Bcl2 induces DNA replication stress by inhibiting ribonucleotide reductase," Cancer Research, vol. 74, no. 1, pp. 212-223, 2014.

[12] H. Tian, C. Ge, H. Li et al., "Ribonucleotide reductase M2B inhibits cell migration and spreading by early growth response protein 1-mediated phosphatase and tensin homolog/Aktl pathway in hepatocellular carcinoma," Hepatology, vol. 59, no. 4, pp. 1459-1470, 2014.

[13] K. M. Aird, H. Li, F. Xin, P. A. Konstantinopoulos, and R. Zhang, "Identification of ribonucleotide reductase M2 as a potential target for pro-senescence therapy in epithelial ovarian cancer," Cell Cycle, vol. 13, no. 2, pp. 199-207, 2014.

[14] H. Y. Kim and C. W. Kim, "Ribonucleotide reductase subunit $\mathrm{m} 2$ can be new molecular target and prognostic biomarker of hepatocellular carcinoma," Gut and Liver, vol. 8, no. 6, pp. 580581, 2014.

[15] B. Lee, S. Y. Ha, D. H. Song, H. W. Lee, S. Y. Cho, and C. Park, "High expression of ribonucleotide reductase subunit $\mathrm{m} 2$ correlates with poor prognosis of hepatocellular carcinoma," Gut and Liver, vol. 8, no. 6, pp. 662-668, 2014.

[16] T. Kimura, S. Takeda, Y. Sagiya, M. Gotoh, Y. Nakamura, and H. Arakawa, "Impaired function of p53R2 in Rrm2b-null mice causes severe renal failure through attenuation of dNTP pools," Nature Genetics, vol. 34, no. 4, pp. 440-445, 2003.

[17] L. Xue, B. Zhou, X. Liu, W. Qiu, Z. Jin, and Y. Yen, "Wildtype 53 regulates human ribonucleotide reductase by proteinprotein interaction with p53R2 as well as hRRM2 subunits," Cancer Research, vol. 63, no. 5, pp. 980-986, 2003.

[18] L. Xue, B. Zhou, X. Liu et al., "Ribonucleotide reductase small subunit p53R2 facilitates p21 induction of G1 arrest under UV irradiation," Cancer Research, vol. 67, no. 1, pp. 16-21, 2007.
[19] X. Liu, L. Xue, and Y. Yen, "Redox property of ribonucleotide reductase small subunit M2 and p53R2," Methods in Molecular Biology, vol. 477, pp. 195-206, 2008.

[20] X. Liu, B. Zhou, L. Xue et al., "Metastasis-suppressing potential of ribonucleotide reductase small subunit p53R2 in human cancer cells," Clinical Cancer Research, vol. 12, no. 21, pp. 63376344, 2006.

[21] L. Chang, R. Guo, Q. Huang, and Y. Yen, "Chromosomal instability triggered by Rrm $2 \mathrm{~b}$ loss leads to IL-6 secretion and plasmacytic neoplasms," Cell Reports, vol. 3, no. 5, pp. 1389-1397, 2013.

[22] S. Yanamoto, G. Kawasaki, I. Yoshitomi, and A. Mizuno, "Expression of p53R2, newly p53 target in oral normal epithelium, epithelial dysplasia and squamous cell carcinoma," Cancer Letters, vol. 190, no. 2, pp. 233-243, 2003.

[23] N.-Y. Hsu, J.-Y. Wu, X. Liu et al., "Expression status of ribonucleotide reductase small subunits hRRM2/p53R2 as prognostic biomarkers in stage I and II non-small cell lung cancer," Anticancer Research, vol. 31, no. 10, pp. 3475-3481, 2011.

[24] X. Liu, L. Lai, X. Wang et al., "Ribonucleotide reductase small subunit M2B prognoses better survival in colorectal cancer," Cancer Research, vol. 71, no. 9, pp. 3202-3213, 2011.

[25] X. Wang, X. Liu, L. Xue et al., "Ribonucleotide reductase subunit p53R2 regulates mitochondria homeostasis and function in $\mathrm{KB}$ and PC-3 cancer cells," Biochemical and Biophysical Research Communications, vol. 410, no. 1, pp. 102-107, 2011.

[26] K. Zhang, J. Wu, X. Wu et al., "p53R2 inhibits the proliferation of human cancer cells in association with cell-cycle arrest," Molecular Cancer Therapeutics, vol. 10, no. 2, pp. 269-278, 2011.

[27] E. C. Cho, M. L. Kuo, X. Liu et al., "Tumor suppressor FOXO3 regulates ribonucleotide reductase subunit RRM2B and impacts on survival of cancer patients," Oncotarget, vol. 5, no. 13, pp. 4834-4844, 2014.

[28] M.-L. Kuo, A. J. Sy, L. Xue et al., "RRM2B suppresses activation of the oxidative stress pathway and is Up-regulated by P53 during senescence," Scientific Reports, vol. 2, article 822, 2012.

[29] M. Y. Kang, H.-B. Kim, C. Piao et al., "The critical role of catalase in prooxidant and antioxidant function of p53," Cell Death and Differentiation, vol. 20, no. 1, pp. 117-129, 2013.

[30] E.-C. Cho, S. Zheng, S. Munro et al., "Arginine methylation controls growth regulation by E2F-1," The EMBO Journal, vol. 31, no. 7, pp. 1785-1797, 2012.

[31] M. Jansson, S. T. Durant, E.-C. Cho et al., "Arginine methylation regulates the p53 response," Nature Cell Biology, vol. 10, no. 12, pp. 1431-1439, 2008.

[32] S. Sandoval, C. Kraus, E.-C. Cho et al., "Sox4 cooperates with CREB in myeloid transformation," Blood, vol.120, no. 1, pp. 155$165,2012$.

[33] M. Pigazzi, E. Manara, S. Bresolin et al., "MicroRNA-34b promoter hypermethylation induces CREB overexpression and contributes to myeloid transformation," Haematologica, vol. 98, no. 4, pp. 602-610, 2013.

[34] S. Wu, M. J. Sampson, W. K. Decker, and W. J. Craigen, "Each mammalian mitochondrial outer membrane porin protein is dispensable: effects on cellular respiration," Biochimica et Biophysica Acta, vol. 1452, no. 1, pp. 68-78, 1999.

[35] I. Roman, J. Figys, G. Steurs, and M. Zizi, "In vitro interactions between the two mitochondrial membrane proteins VDAC and cytochrome $c$ oxidase," Biochemistry, vol. 44, no. 39, pp. 1319213201, 2005. 
[36] S. A. Siletsky and A. A. Konstantinov, "Cytochrome c oxidase: charge translocation coupled to single-electron partial steps of the catalytic cycle," Biochimica et Biophysica Acta, vol. 1817, no. 4, pp. 476-488, 2012.

[37] P. H. Boer and M. W. Gray, "Genes encoding a subunit of respiratory NADH dehydrogenase (ND1) and a reverse transcriptase-like protein (RTL) are linked to ribosomal RNA gene pieces in Chlamydomonas reinhardtii mitochondrial DNA," The EMBO Journal, vol. 7, no. 11, pp. 3501-3508, 1988.

[38] K. A. Roebuck, "Oxidant stress regulation of IL-8 and ICAM-1 gene expression: differential activation and binding of the transcription factors AP-1 and NF-kappaB (Review)," International Journal of Molecular Medicine, vol. 4, no. 3, pp. 223-230, 1999.

[39] S. Yanagisawa, A. Koarai, H. Sugiura et al., "Oxidative stress augments toll-like receptor 8 mediated neutrophilic responses in healthy subjects," Respiratory Research, vol. 10, article 50, 2009.

[40] D. Laveti, M. Kumar, R. Hemalatha et al., "Anti-inflammatory treatments for chronic diseases: a review," Inflammation \& Allergy-Drug Targets, vol. 12, no. 5, pp. 349-361, 2013.

[41] A. Sanchez, D. Tripathy, X. Yin et al., "p38 MAPK: a mediator of hypoxia-induced cerebrovascular inflammation," Journal of Alzheimer's Disease, vol. 32, no. 3, pp. 587-597, 2012.

[42] J. Cheriyan, A. J. Webb, L. Sarov-Blat et al., "Inhibition of p38 mitogen-activated protein kinase improves nitric oxide-mediated vasodilatation and reduces inflammation in hypercholesterolemia," Circulation, vol. 123, no. 5, pp. 515-523, 2011.

[43] J. Bauer, S. Namineni, F. Reisinger, J. Zller, D. Yuan, and M. Heikenwlder, "Lymphotoxin, NF- $\kappa \mathrm{B}$, and cancer: the dark side of cytokines," Digestive Diseases, vol. 30, no. 5, pp. 453-468, 2012.

[44] F. G. Osorio, C. López-Otín, and J. M. P. Freije, "NF- $\kappa$ B in premature aging," Aging, vol. 4, no. 11, pp. 726-727, 2012.

[45] J. Tobon-Velasco, E. Cuevas, and M. Torres-Ramos, "Receptor for AGEs (RAGE) as mediator of NF-kB pathway activation in neuroinflammation and oxidative stress," CNS \& Neurological Disorders-Drug Targets, vol. 13, no. 9, pp. 1615-1626, 2014.

[46] T. Zarubin and J. Han, "Activation and signaling of the p38 MAP kinase pathway," Cell Research, vol. 15, no. 1, pp. 11-18, 2005.

[47] X. Wang, A. Zhenchuk, K. G. Wiman, and F. Albertioni, "Regulation of p53R2 and its role as potential target for cancer therapy," Cancer Letters, vol. 276, no. 1, pp. 1-7, 2009.

[48] M. H. Tsai, X. Chen, G. V. R. Chandramouli et al., "Transcriptional responses to ionizing radiation reveal that $\mathrm{p} 53 \mathrm{R} 2$ protects against radiation-induced mutagenesis in human lymphoblastoid cells," Oncogene, vol. 25, no. 4, pp. 622-632, 2006.

[49] K. H. Vousden, "Partners in death: a role for $\mathrm{p} 73$ and NF- $\kappa \mathrm{B}$ in promoting apoptosis," Aging, vol. 1, no. 3, pp. 275-277, 2009.

[50] T. Lawrence, "The nuclear factor NF-kappaB pathway in inflammation," Cold Spring Harbor Perspectives in Biology, vol. 1, no. 6, Article ID a001651, 2009. 


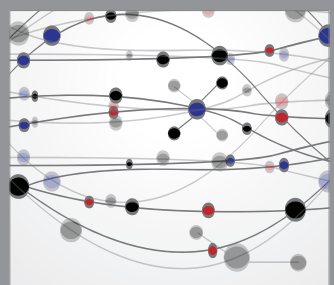

The Scientific World Journal
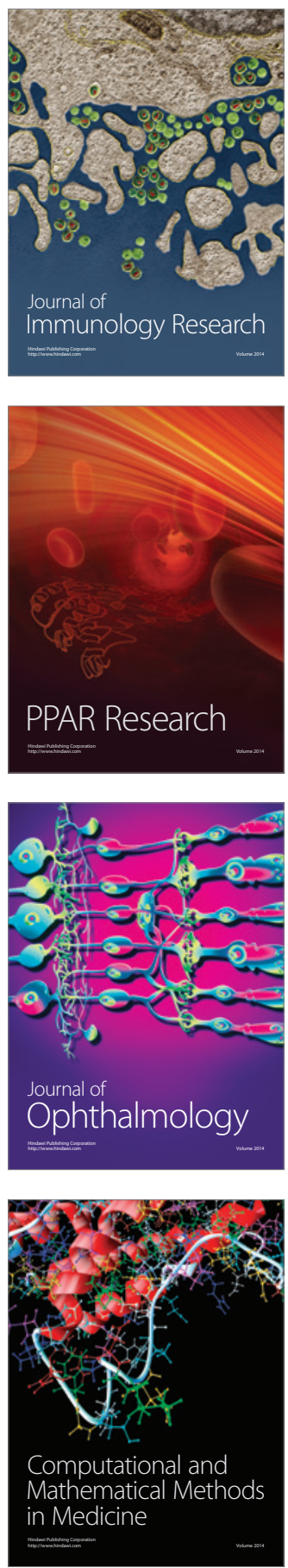

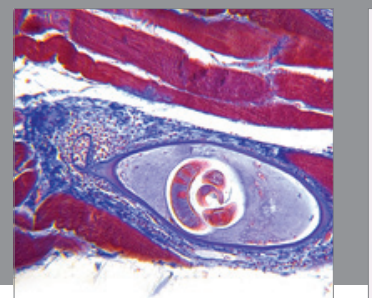

Gastroenterology

Research and Practice
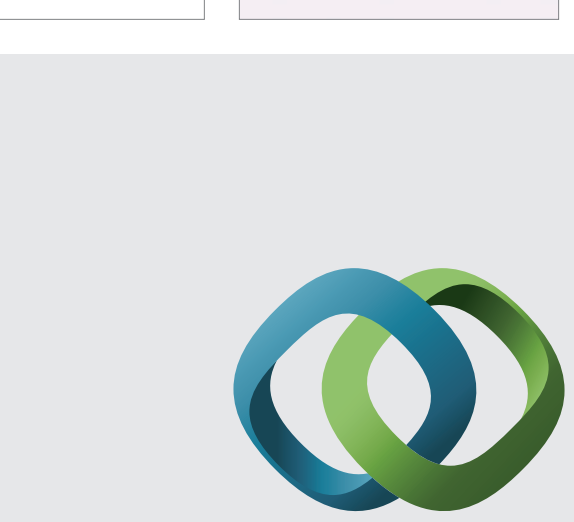

\section{Hindawi}

Submit your manuscripts at

http://www.hindawi.com
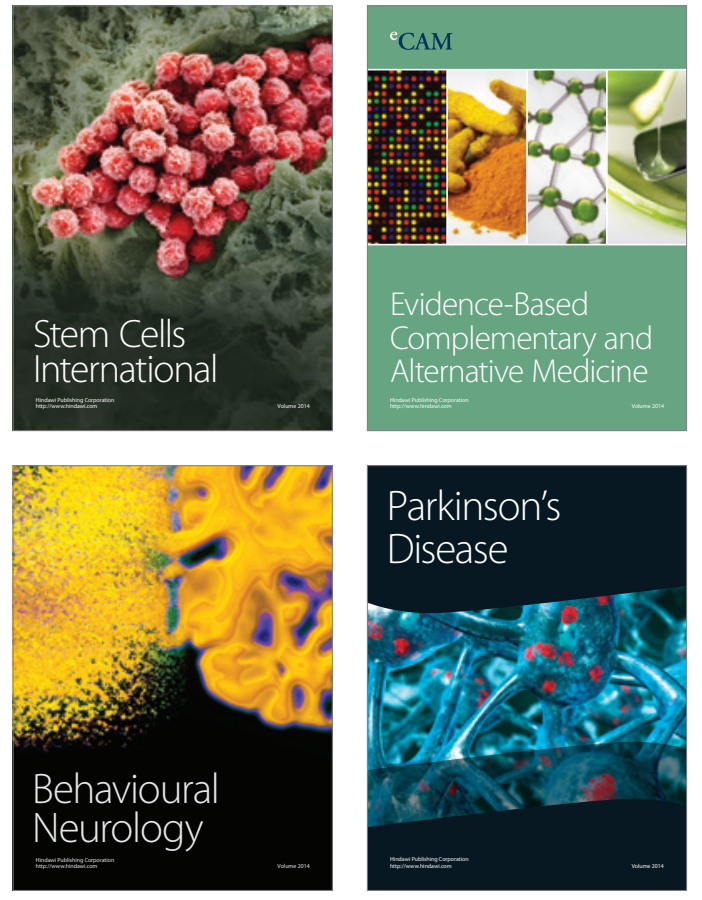
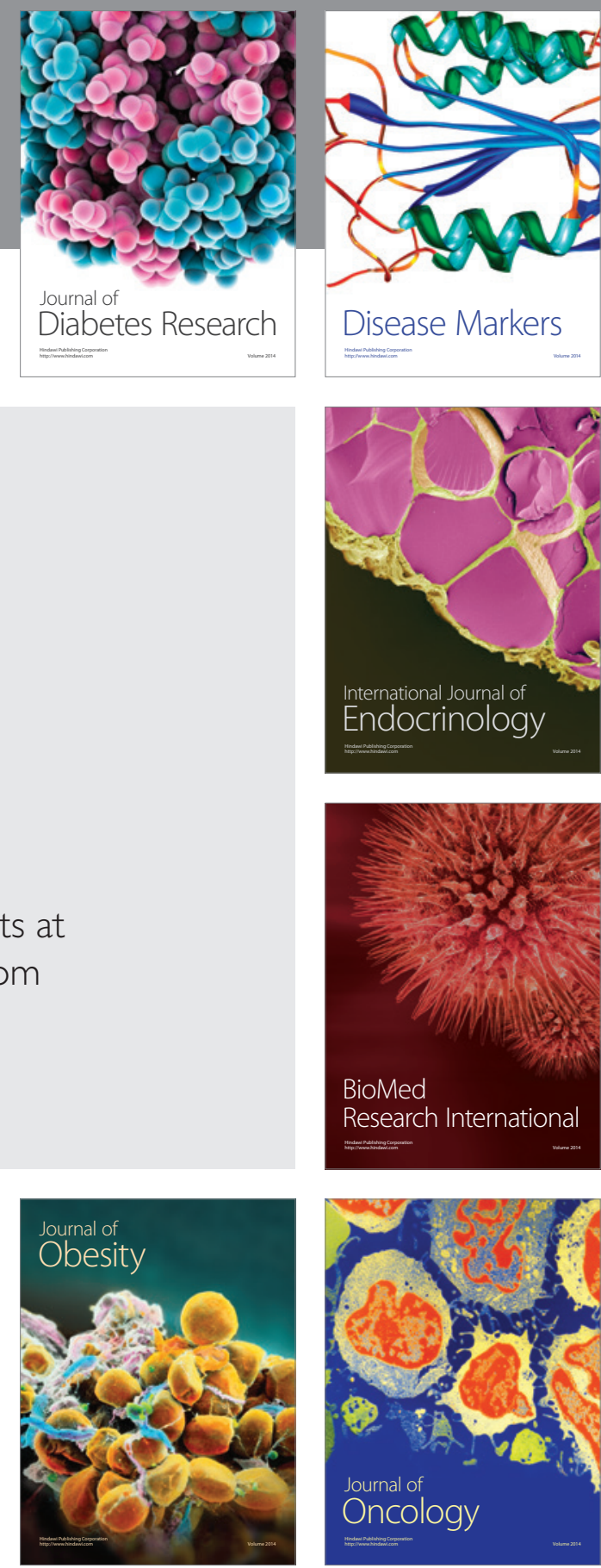

Disease Markers
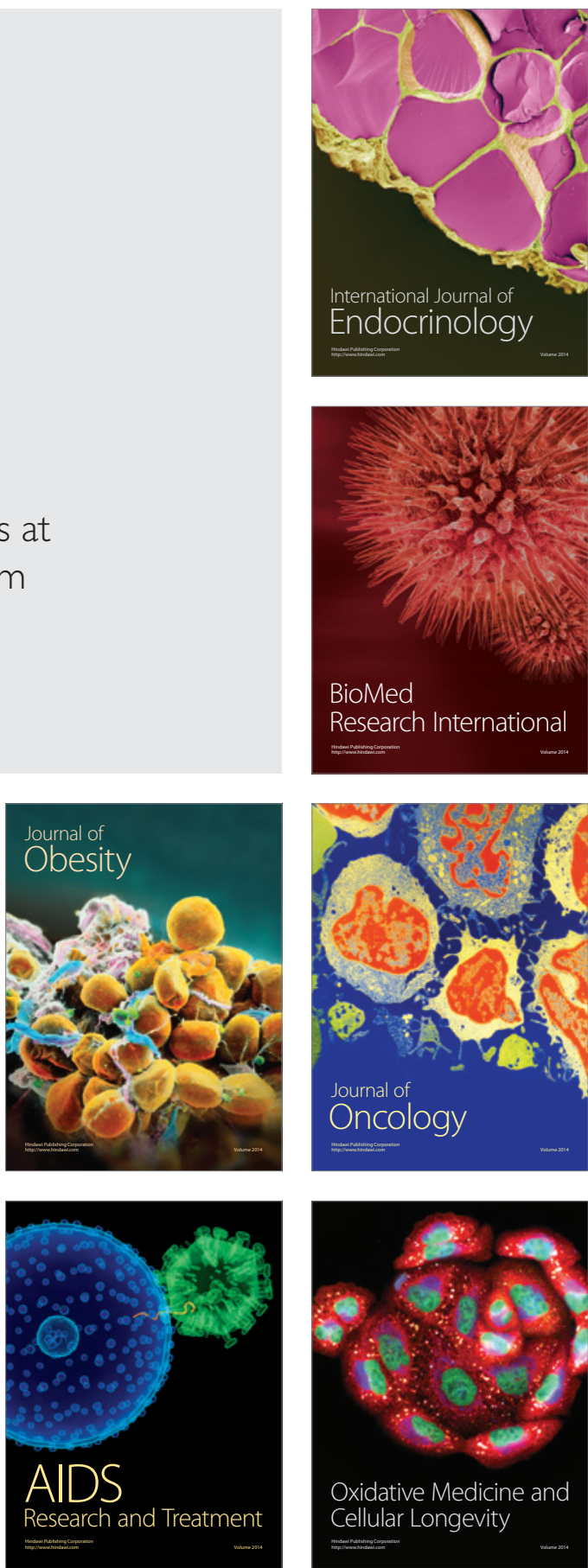